\title{
Root Acclimatization of the Micropropagated Fruit Tree Rootstock 'Adafuel' (Prunus dulcis (Mill.) D.A. Webb x P. persica (L.) Batsch)
}

\author{
Juan A. Marin, Arancha Arbeloa, Marta Castillo and Pilar Andreu \\ Estacion Experimental de Aula Dei (CSIC) \\ Apartado 202, 50080 Zaragoza, Spain \\ e-mail:jmarin@eead.csic.es
}

Keywords: Root anatomy, in vitro culture, histochemistry, exodermis, endodermis, Casparian strips, phi-thickenings

\begin{abstract}
Micropropagated plants undergo acclimatization to adapt to the greenhouse environment. In vitro grown plants have a modified anatomy caused by the in vitro environment. The adaptive capacity of plants (phenotypic plasticity) is based on the modification of their anatomical structure and physiology. This adaptation has been demonstrated in studies dealing with leaf tissues of different species. However, little attention has been paid to root changes and to its capacity to adapt to acclimatization. In this work, changes in roots of in vitro rooted shoots of the almond $x$ peach hybrid rootstock 'Adafuel' (Prunus dulcis $x$ persica) during acclimatization are described using histological and histochemical techniques. Root structure of in vitro rooted shoots was compared with that of acclimatized shoots as well as with that of in vivo rooted shoots, that displayed normal anatomy and functioning. Normal roots, grown under in vivo conditions, showed well defined phloem and xylem vessels, endodermis with frequent presence of Casparian strips and phithickenings in the cell walls of the cortex close to the endodermis. These characters match with a functional structure able to supply water to the aerial part of the plant and related to a selective nutrient uptake. On the other hand, roots formed in vitro lacked well defined vessels and showed weakly stained Casparian strips and the absence of phi-thickenings, which agrees with a limited water transport. Nevertheless, in vitro formed roots were able to adapt to the new environment and undertake diverse changes during the acclimatization of micropropagated plants.
\end{abstract}

\section{INTRODUCTION}

Micropropagated plants must experience an acclimatization process to be able to stand ex vitro conditions. In vitro grown plants display anatomical modifications due to the environmental conditions faced, mainly low light intensity and high relative humidity, that originates excessive water transpiration and desiccation when exposed to low relative humidity. Nevertheless, plants can adapt to the new environment thanks to their phenotypic plasticity.

Anatomical and physiological changes during acclimatization have been studied previously (see Pospíšilová et al., 1999 for a review), but these studies dealt mainly with the aerial part of the plant. Thus, whereas leaves have received a remarkable attention, roots, the "hidden half", have remained unattended. Roots are important in fruit tree rootstocks, since unlike scions, roots remain after grafting to support tree growth. In addition, micropropagation is the method of choice in many cases for fruit-tree rootstocks. 
In this work we describe root changes during acclimatization using histological and histochemical techniques. Root structure of in vitro rooted shoots was compared with that of acclimatized shoots as well as with that of in vivo rooted shoots that displayed normal anatomy and functioning.

\section{MATERIALS AND METHODS}

Shoots of the almond x peach hybrid rootstock 'Adafuel' (Prunus dulcis (Mill.) D.A. Webb $x$ P. persica (L.) Batsch) introduced in vitro more than one year ago, were used. They were multiplied in a MS modified medium (Murashige and Skoog, 1962) with IBA $(0.5 \mu \mathrm{M})$ and BAP $(5 \mu \mathrm{M})$, and with sucrose $(30 \mathrm{~g} / \mathrm{L})$ and Difco-Bacto agar $(7 \mathrm{~g} / \mathrm{L})$. Micropropagated shoots were rooted in vitro in a similar medium but with MS salts reduced to a half, without $\mathrm{BAP}$, and with IBA increased to $5 \mu \mathrm{M}$. In vivo rooting was induced by dipping $30 \mathrm{~s}$ the base of micropropagated shoots in an aqueous solution of IBA $(1 \mathrm{mM})$, then shoots were allowed to root under high relative humidity in Jiffy pots with a peat:vermiculite $(1: 1)$ substrate. Shoots (in vivo and in vitro) were acclimatized after root formation in a plastic tunnel in a greenhouse with the same substrate under daily increasing exposures to low relative humidity (Marín, 2003).

Roots of about $3 \mathrm{~cm}$ length from at least three different plants per treatment were studied. Free hand root sections were made with a razor blade and placed in slides with different fluorescence stains: Calcofluor (aqueous solution) at $0.7 \%$ for cellulose (Hughes and McCully, 1975), Auramine $\mathrm{O}$ at $0.01 \%$ in $0.05 \mathrm{M}$ phosphate buffer for lipids and suberin (Heslop-Harrison, 1977), and Aniline Blue (aqueous solution) at $0.1 \%$ for callose (Martin, 1959) and to enhance lignin autofluorescence. An epifluorescent Zeiss microscope was used and the slides were observed with filters for UV light (Calcofluor and Aniline Blue) or for Blue light (Auramine O).

\section{RESULTS AND DISCUSSION}

In vitro roots maintain the general structure of the root, in spite of the presence of differences between in vitro and normal roots that are summarized in Table 1. Roots showed different developmental zones: the root cap and the meristematic zone, the elongation zone, and the maturation zone, following Taiz and Zeiger (2002) terminology, but there were differences in their histological structure depending on the treatment. This structure was formed by an external layer of cells (epidermis) with root hairs in the maturation zone that later became less apparent or disappeared, the cortex that is limited by the exodermis in the outer part next to the epidermis and the endodermis in the inner part next to the stele, and the vascular cylinder or stele that contains the phloem and the xylem.

\section{In vitro roots}

Roots formed in vitro showed phloem and xylem vessels with few elements and small size (Fig. 1A) and an epidermis with small hairs. In addition, less developed Casparian strips, both in the exodermis and in the endodermis, can be observed. These facts agree with a limited water absorption and transport as is expected under in vitro conditions. In vitro roots do not need carbohydrates from the shoot to grow inside the culture medium, and, on the other hand, shoots, with a limited transpiration inside the culture vessel, can continue the absorption of water from medium. Casparian strips are related with a selective absorption of nutrients, preventing the apoplastic transport of substances both from the soil to the shoot and vice versa (Taiz and Zeiger, 2002). It seems 
that under in vitro conditions root tissues do not mature as in normal conditions, and thus, root hairs and xylem vessels are less represented than in normal conditions, according to their limited functioning. This emphasizes the role of the acclimatization process that allows the adaptation of root tissues to water absorption and transport.

In vitro roots showed the absence of phi-thickenings in the cortex. Phi-thickenings are lignified wall thickenings of cortical cells of certain gymnosperms and a few species of angiosperms as the Rosaceae (Peterson et al., 1981; Gerrath et al., 2002; Evert, 2006) and their role in root physiology is not well established yet. Here, the presence of phithickening in both in vivo roots and in vitro roots after acclimatization, together to their absence in in vitro roots, suggests that their role can be related to water transport and root functioning. Moreover, phi-thickenings are present only in the maturation zone (Soukup et al., 2004), where simultaneously appeared root hairs and mature xylem vessels.

In vitro cortical cells showed, however, abundant lipid deposits strongly stained with Auramine O (Fig. 1A) that are present in the other treatments in a lesser extent. Since in vitro roots can absorb nutrients directly from the culture medium, the presence of such abundant lipid deposits could be related with cellular metabolism.

\section{In vivo roots}

Normal roots, grown under in vivo conditions, showed at the maturation zone well defined phloem and xylem vessels in the vascular cylinder (Fig. 1B), frequent presence of Casparian strips in both endodermis and exodermis, and phi-thickenings in the cell walls of the cortex close to the endodermis. These characters match with a functional structure able to supply water to the aerial part of the plant and related to a selective nutrient uptake by blocking the apoplastic transport (Taiz and Zeiger, 2002).

\section{Acclimatized roots}

During acclimatization, in vitro formed roots were able to adapt to the new environment both by forming new root tissues and by undertaking different changes in the root structure of older tissues. After a 2 weeks period of acclimatization under greenhouse conditions, new leaves were formed and roots became functional by acquiring both an anatomical structure like that of the in vivo formed roots, and the capacity to supply water to compensate the water lost by transpiration in the aerial part of the plant.

From the tip to the base, roots displayed different structures. After a simplified start elongation zone, where the vascular cylinder is little represented, the stele is becoming larger and the phloem and xylem vessels increased in both number and size of the elements. At the maturation zone, roots showed an epidermis with long and abundant root hairs and well formed xylem and phloem vessels (Fig. 1C). The endodermis and exodermis were well developed and Casparian strips were observed in both cell layers. These changes in root structure agree with an efficient water transport to the shoot and with a normal root functioning. In addition, phi-thickenings were formed in the cortical cells close to the endodermis. Soukup et al. (2004) described a similar phenomenon in wild cherry where phi-thickenings appeared after acclimatization (they did not studied in vivo formed roots), but they did not found differences after acclimatization in other characters. After root maturation, secondary roots can appear from the stele, and in the base of the root, the vascular cylinder contained thicker and highly lignified xylem vessels protected by suberinized cell layers while the cortical cells showed little intercellular adherence. 
Root development differences between in vitro and in vivo roots can be explained in terms of the important differences that exist between in vitro and greenhouse environments. In vitro roots must grow through a highly hydrated gelled medium that contains exogenous auxins and sucrose in large amounts. Since the agar-gelled medium is translucent, it transmits light from the chamber that is known to have an inhibitory effect on rooting (Drew, 1988). The exposure to auxin can induce ethylene formation that, in turn, affects root formation (Coleman et al, 1980). Sucrose favours root growth as a source of both carbon and energy (see George, 1996, for a review), however, this beneficial effect might prevent roots from being natural sink organs of carbohydrates from the shoot. In addition, gas exchange is reduced and an oxygen deficit may happen, although the depth of the culture medium is not too large and some oxygen can diffuse reaching the roots (Crane and Hughes, 1990). On the other hand, in vivo roots are formed in an opaque and well aerated substrate without any external source of sugar and auxins, therefore, roots are dependent on the shoot to obtain the necessary nutrients and energy to grow. As a compensation, water uptake from the roots will soon originate a water flow to the shoot. The role of root hairs in acclimatized in vitro roots, lost after the plant removal from culture medium, would be resumed as soon as root growth is active again and an intimate contact with soil particles is established.

\section{CONCLUSIONS}

This work has studied the morphological changes that in vitro formed roots experience during the acclimatization process. These changes are in accordance with the morphology of a normal root formed under in vivo conditions and are related with water transport, since they mainly affect to xylem and phloem vessels, the endodermis with their apoplastic barrier, and the cortical cell wall modifications known as phi-thickenings. Thus, these changes suggest that in vitro roots are unable to provide water supply to the shoot of micropropagated plants, being necessary to accomplish a simultaneous acclimatization with that of the aerial part.

Here, we have described the phenotypic plasticity of in vitro grown roots. Data presented here support the fundamental role that roots play during acclimatization, since they change during the process from a non-functional structure to a functional one that resembles that formed in normal in vivo conditions.

\section{Literature cited}

Coleman W.R., Huxter, T.J., Reid, D.M. and Thorpe, T.A. 1980. Ethylene as an endogenous inhibitor of root regeneration in tomato leaf discs cultured in vitro. Physiol. Plant. 48: 519-525.

Crane, J. and Hughes, H. 1990. Medium overlays for improved hardening of micropropagated potatoes. HortScience 25:794-795.

Drew, R.A. 1988. Rapid clonal propagation of papaya in vitro from mature field-grown trees. HortScience 23: 609-611.

Evert, R.F. 2006. Esau's Plant anatomy: meristems cells, and tissues of the plant body: their structure, function, and development. Wiley \& Sons, Inc. Hoboken.

George, E.F. 1996. Plant propagation by tissue culture. Part 2. In practice. Exegetics Limited, Edington.

Gerrath, J.M., Covington L., Doubt, J. and Larson, D.W. 2002. Occurrence of phi thickenings is correlated with gymnosperm systematics. Can. J. Bot. 80: 852-860. 
Heslop-Harrison, Y. 1977. The pollen stigma interaction: pollen-tube penetration in Crocus. Ann. Bot. 41: 913-922.

Hughes, J. and McCully, M.E. 1975. The use of an optical brightener in the study of plant structure. Stain Tech. 50: 319.

Marín, J.A. 2003. High survival rates during acclimatization of micropropagated fruit tree rootstocks by increasing exposures to low relative humidity. Acta Hort. 616: 139-142.

Martin, F.W. 1959. Staining and observing pollen tubes in the style by means of fluorescence. Stain Tech. 34: 128-128.

Murashige, T. and Skoog, F. 1962. A revised medium for rapid growth and bioassays with tobacco tissue culture. Physiol. Plant. 15: 473-497.

Peterson, C.A., Emanuel, M.E. and Weerdenburg, C.A. 1981. The permeability of phi thickenings in apple (Pyrus malus) and geranium (Pelargonium hortorum) roots to an apoplastic fluorescent dye tracer. Can. J. Bot. 59: 1107-1110.

Pospíšilová, J., Tichá, I., Kadleček, P., Haisel, D. and. Plzáková, Š. 1999. Acclimatization of micropropagated plants to ex vitro conditions. Biol. Plant. 42: 481-497.

Soukup, A., Mala, J., Hrubcova, M., Kalal, J., Votrubova, O. and Cvikrova, M. 2004. Differences in anatomical structure and lignin content of roots of pedunculate oak and wild cherry-tree plantlets during acclimation. Biol. Plant. 48: 481-489.

Taiz, L. and Zeiger, E. 2002. Plant Physiology. Sinauer Associates, Inc. Sunderland. 


\section{Tables}

Table 1. Comparison of the main histological characters observed.

\begin{tabular}{llllll}
\hline Roots & $\begin{array}{l}\text { root hairs } \\
\text { (epidermis) }\end{array}$ & $\begin{array}{l}\text { lipid } \\
\text { cellular } \\
\text { content } \\
\text { (cortex) }\end{array}$ & $\begin{array}{l}\text { Casparian } \\
\text { strips } \\
\text { (endodermis, } \\
\text { exodermis) }\end{array}$ & $\begin{array}{l}\text { phi- } \\
\text { thickenings }\end{array}$ & $\begin{array}{l}\text { vascular } \\
\text { cylinder }\end{array}$ \\
\hline in vitro & short & abundant & $\begin{array}{l}\text { weak lignin } \\
\text { and suberin } \\
\text { stain }\end{array}$ & absent & $\begin{array}{l}\text { not well } \\
\text { developed }\end{array}$ \\
\hline in vivo & long & rare & strong stain & present & $\begin{array}{l}\text { well } \\
\text { developed }\end{array}$ \\
\hline $\begin{array}{l}\text { in vitro/ } \\
\text { acclimatized }\end{array}$ & long & rare & strong stain & present & $\begin{array}{l}\text { well } \\
\text { developed }\end{array}$ \\
\hline
\end{tabular}

Figures
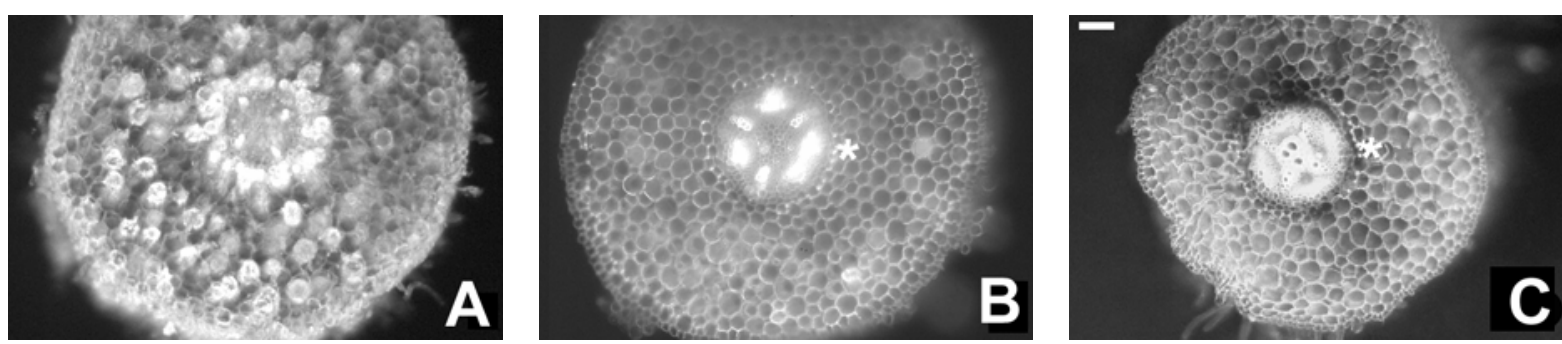

Fig. 1. Transversal sections of the roots of A) in vitro root showing abundant cellular deposits of lipids and an immature vascular system, B) in vivo root showing well developed xylem and phloem vessels as well as phi-thickenings in cortical cells close to the stele $(*)$, and $\mathrm{C}$ ) in vitro root after acclimatization showing phloem and xylem vessels, phi-thickenings $(*)$ and root hairs (bar: $100 \mu \mathrm{m}$ ). 